\title{
Nicotine Replacement Therapy Use Predicts Smoking and Drinking Outcomes among Heavy-Drinking Smokers Calling a Tobacco Quitline
}

\author{
Alana M. Rojewski, ${ }^{1,2}$ Lisa M. Fucito, ${ }^{1}$ Stephen Baldassarri, ${ }^{3}$ Andrew Hyland, ${ }^{4}$ \\ K. Michael Cummings, ${ }^{5}$ and Benjamin A. Toll ${ }^{1,2,6,7}$ \\ ${ }^{1}$ Department of Psychiatry, Yale School of Medicine, New Haven, Connecticut \\ 2 Department of Public Health Sciences, Medical University of South Carolina, Charleston, South Carolina \\ ${ }^{3}$ Department of Internal Medicine, Yale School of Medicine, New Haven, Connecticut \\ ${ }^{4}$ Roswell Park Cancer Institute, Buffalo, New York \\ ${ }^{5}$ Department of Psychiatry \& Behavioral Sciences, Medical University of South Carolina, Charleston, South Carolina \\ ${ }^{6}$ Yale Cancer Center, New Haven, Connecticut \\ 7 Smilow Cancer Hospital at Yale-New Haven, New Haven, Connecticut
}

\begin{abstract}
ntroduction: Suboptimal use of nicotine replacement therapy (NRT) may pose a significant barrier to smokers attempting to quit. We examined NRT use as a predictor of smoking abstinence and heavy drinking in a randomised trial of hazardous drinkers who contacted the NY State Smokers' quitline for smoking cessation assistance.

Methods: Participants $(N=1,948)$ received either Tobacco Only Counselling or Alcohol + Tobacco Counselling (ATC), both in addition to a 2-week supply of NRT. NRT use, smoking status, and heavy drinking days were assessed by self-report at the 7-month follow-up.

Results: Of those smokers who completed the 7-month follow-up ( $N=843), 53.1 \%$ used all of the NRT and $40.6 \%$ used some. Those who used all of the NRT were more likely to be abstinent from smoking than those who used some, and more likely to report no heavy drinking days than those who used some or none.

Conclusions: Approximately half of the heavy drinking smokers calling the quitline are willing to use the 2-week supply of free NRT, and most will at least try it. Those who reported using all of the NRT were more likely to report smoking abstinence and no heavy drinking days at the 7-month follow-up.
\end{abstract}

\section{Introduction}

Despite a significant decline in the number of adult smokers in the US over the past 50 years, approximately $18 \%$ of the US population continues to smoke (Fiore, Schroeder, \& Baker, 2014). A recent meta-analysis of the existing evidence found that 6 month smoking cessation rates reached a maximum of only $31.5 \%$ with the use of combination NRT (nicotine patch plus inhaler; Cahill, Stevens, \& Lancaster, 2014). Among the many potential barriers to quitting smoking, suboptimal use of NRT may be hampering quit attempts. Many public programs (e.g., state quitlines) offer free 2-week supplies of NRT. In an analysis of 4 regional tobacco control programs in New York State, $78 \%-89 \%$ of people who received NRT reported use of the medication. The average number of days of patch use was 9 days ( $64 \%$ of available days) for those who received a 2-week supply (Cummings et al., 2006). Thus, many smokers will use the NRT, and some will not use the medication at all. Further, some may try the NRT for a few days and discontinue use.

Several studies have documented the effect of premature discontinuation of NRT and smoking quit rates. For example, Balmford and colleagues found that approximately one third of smokers prematurely discontinued NRT within less than 2 weeks of use and were less likely to achieve 6 month continuous abstinence compared to those who completed the NRT regimen (Balmford, Borland, Hammond, \& Cummings, 2011). The two most 
common reasons given for discontinuation were relapse back to smoking and perceived side effects, a finding that was also reported by Burns and Levinson (2008). Another secondary analysis of data from a randomised trial evaluating the efficacy of an over-the-counter nicotine patch versus placebo found that adherence to NRT (defined as wearing the patch for $\geq 20$ days) within the first 3 weeks of treatment was associated with a longer duration of abstinence (Shiffman, Sweeney, Ferguson, Sembower, \& Gitchell, 2008).

Among smokers, use of NRT may be particularly problematic in patients who also consume alcohol. For example, a cohort analysis of medication use among veterans attending primary care clinics demonstrated that higher scores on a hazardous alcohol use screening tool (AUDIT-C) were associated with increased risk for hypertension and hyperlipidemia medication non-adherence (Banta et al., 2009). Further, a study of veterans who self-reported alcohol consumption demonstrated a doseresponse and temporal association with non-adherence to medications (Braithwaite et al., 2005). Specifically, binge drinkers missed doses on $11.0 \%$ of drinking days, $7.0 \%$ of post-drinking days, and $4.1 \%$ of non-drinking days. Thus, heavy alcohol consumption may correspond to a higher risk for non-adherence or suboptimal use of NRT among heavy drinking smokers.

This is a secondary data analysis of a previously conducted randomised-clinical trial (Toll et al., 2015). In the present study, we examine whether use of NRT predicts smoking quit rates and heavy drinking rates in a randomised trial of hazardous drinkers who contacted the NY State Smokers' quitline (NYSSQL) for smoking cessation assistance. We hypothesise that greater use of NRT would be associated with improved rates of smoking cessation and a reduction in the number of heavy drinking days in hazardous drinkers calling the quitline. We also conduct and present exploratory analyses to better understand participants' reasons for not using NRT.

\section{Materials and Methods \\ Participants}

To be eligible, participants had to (1) be a New York State resident at least 18 years of age, (2) speak English, (3) call the NYSSQL for assistance in quitting smoking, (4) not be enrolled in any cessation programs through another source (e.g., their insurance company), and (5) meet hazardous drinking criteria as defined by the National Institute on Alcohol Abuse and Alcoholism (NIAAA). Hazardous drinking was defined as exceeding sex-specific weekly limits (14 drinks/men, 7 drinks/women) or meeting/exceeding daily drinking limits ( 5 drinks/men and 4 drinks/women) at least once in the past year (National Institute on Alcohol Abuse Alcoholism, 2005).

\section{Procedure}

Eligible participants were randomised $(N=1,948)$ to receive either Tobacco Only Counselling (TOC - standard quitline treatment) or ATC. Phone-based counselling was provided at intake and at the quit day follow-up call, which was scheduled for within 30 days of the initial call. The TOC counselling protocol focused on standard smoking cessation advice (i.e., practical counselling - general problem solving and skills training). The ATC counselling protocol focused on having the caller examine how smoking and drinking are paired and the ways in which drinking may have inhibited previous smoking cessation attempts. Callers in both conditions received the "Break Loose!" booklet, a self-help smoking cessation guide, as part of standard care treatment. ATC participants received "Rethinking Drinking," a booklet designed to help people reduce their risk for alcohol problems by analysing their drinking and offering research-based information on the most effective ways to cut down. TOC participants received an additional National Cancer Institute smoking cessation booklet, "Clearing the Air," that describes tools that can help smokers stop smoking and the problems to expect when they quit. In addition to the booklets and counselling, participants received a 2 -week supply of NRT patches free of charge, if medically eligible (e.g., patients who were breastfeeding were not sent any NRT). If participants indicated a medical complication with patches (e.g., skin sensitivity to plastics), and were eligible through their personal insurance or met special qualifying criteria for other state funded programs for gum or lozenge, then they would be offered gum or lozenge in lieu of patches. Complete study details can be found in the primary publication (Toll et al., 2015). Follow-up data were collected at 7 months.

In the primary study, participants in each treatment group completed an equivalent number of counselling sessions (Toll et al., 2015). All participants completed the first counselling sessions at intake, and the second counselling session at the Quit Day Follow-up was completed by $24 \%$ of both the ATC and TOC groups. ATC was associated with a significantly higher rate of smoking abstinence at 7-month follow-up (13.5\%) as compared with TOC (10.3\%). Among participants in the ATC group, participants who did not report any heavy drinking (30.2\%) were significantly more likely to quit smoking than those who reported any heavy drinking (16.8\%).

\section{Data Analysis}

All analyses were conducted with IBM SPSS Statistics (IBM, 2013). The primary outcome in this study was smoking abstinence at the 7-month follow-up and defined as self-reported point prevalence abstinence (not even a puff in the past 7 days). NRT use was assessed by self-report at the 7-month follow-up. Participants were asked to select which category best described their level of medication use: (1) all of it, (2) about half of it, (3) less than half of it, or (4) none of it. NRT use was coded as follows: used all NRT $=1$, used some NRT $=2$ (collapsed half and less than half together), used none $=3$. The secondary outcome was heavy drinking, defined as more than 
Table 1

Relationship of NRT usage to demographic, smoking-related, and alcohol use variables

\begin{tabular}{|c|c|c|c|c|}
\hline Variables & Used All NRT ( $n=448)$ & Used Some NRT ( $n=342)$ & Did Not Use NRT $(n=53)$ & $p$ \\
\hline$\%$ white & 61.6 & 63.2 & 67.9 & 0.65 \\
\hline$\%$ male & 58.5 & 50.3 & 47.2 & $0.04^{*}$ \\
\hline$\%$ with more than a high school degree & 49.3 & 47.4 & 54.7 & 0.59 \\
\hline No. of cigarettes smoked per day & $20.8 \pm 9.9$ & $19.9 \pm 9.2$ & $21.7 \pm 10.4$ & 0.35 \\
\hline No. of years smoking cigarettes & $22.8 \pm 12.8$ & $21.4 \pm 12.9$ & $26.6 \pm 13.1$ & $0.02^{*}$ \\
\hline Age, y & $43.3 \pm 12.7$ & $42.0 \pm 13.1$ & $48.2 \pm 11.1$ & $0.01^{*}$ \\
\hline$\%$ heavy drinking days & $11.6 \pm 21.6$ & $14.4 \pm 24.9$ & $20.0 \pm 31.2$ & $0.03^{*}$ \\
\hline$\%$ completing second counselling session & 38.4 & 32.5 & 17.0 & $0.01^{*}$ \\
\hline
\end{tabular}

Note. Variables were analysed with $\chi^{2}$ tests for categorical variables and one-way ANOVAs for continuous variables. Means are presented with standard deviations. All statistical tests were 2-sided.

4 drinks per day for men and more than 3 drinks per day for women in the 7 days prior to the 7-month follow-up (National Institute on Alcohol Abuse Alcoholism, 2005). We also compared baseline demographic variables among these groups: race, sex, education, number of cigarettes per day, number of years smoking, age, the percent of days that heavy drinking occurred in the prior 7 days, and percentage who completed the second counselling session.

Logistic regression analyses were conducted to evaluate the effects of NRT adherence on 7-day point prevalence smoking abstinence and heavy drinking days in the past 7 days at the 7-month follow-up. One-way ANOVAs for continuous variables and $\chi^{2}$ tests for categorical variables were used to investigate group differences in demographic, smoking, and alcohol variables (e.g., age, cigarettes per day, percentage of heavy drinking days at baseline, etc.) that might affect NRT use. Due to group differences in variables at baseline, analyses controlled for age, number of years smoking, gender, percent heavy drinking days at baseline, completion of the second counselling session, and treatment condition. Participants who were never mailed NRT (e.g., due to medical ineligibility) were exclude from the responder analyses $(N=10)$, as well as those who reported using NRT but could not remember the amount used $(N=3)$. Missing assessment data at the 7-month follow-up were excluded from analyses as well, leaving a final sample of 843 respondents with complete data ( $43 \%$ response rate). Further, we conducted exploratory analyses of reasons for reported non-use of NRT among the subsample of participants who indicated that they used none of the NRT.

\section{Results}

\section{Participant Demographic and Clinical Characteristics}

Of those smokers who successfully completed the 7-month follow-up assessment $(N=843), 88.7 \%$ received patches and $11.3 \%$ received gum or lozenge. Further, $93.7 \%$ reported using their medication. $53.1 \%$ of participants used the entire two week supply of nicotine medication sent to them $(N=448), 40.6 \%$ used some of the medication $(N$
$=342)$, and $6.3 \%$ used none of the medication $(N=53)$. Participant demographics are presented in Table 1. Those who used all of the NRT were younger $(43.3 \pm 12.7)$, more likely to be male (58.5\%), had been smoking for a fewer number of years $(22.8 \pm 12.8)$, reported a lower percentage of heavy drinking days at baseline (11.6\%), and were more likely to complete the second counselling session (38.4\%). Percentage of heavy drinking days at baseline refers to the percentage of self-reported NIAAA defined heavy drinking days (for men $>4$ a day; for women $>3$ a day) in the week before the baseline assessment was conducted. Race, education level, and number of cigarettes per day were not statistically significant for the different amounts of NRT use.

\section{NRT use and Smoking/Drinking Outcomes}

We tested NRT use as a predictor of smoking quit rates at the 7-month follow-up. As presented in Table 2, those who used all of the NRT were more likely to be abstinent than those who used some (30.8\% vs. $17.8 \%$; Odds Ratio $[\mathrm{OR}]=2.07,95 \%$ Confidence Interval $[\mathrm{CI}]: 1.46-2.93 ; p$ $<0.001)$. The difference in abstinence rates for those who used all NRT (30.8\%) compared to those who used none $(17.0 \%)$ was not statistically significant, even though there was a similar proportion of abstinence with those who used some $(17.8 \%)$. This is likely due to a difference in power, as there were only 53 people who used none of the NRT. In addition, treatment group status and completion of the second counselling session were also statistically significant in this model. Thus, those in the ATC condition were more likely to be abstinent at the 7-month follow-up (OR $=1.44,95 \%$ CI: $1.04-1.99 ; p=0.03)$, as well as those participants who completed the Quit Day Follow-up call $(\mathrm{OR}=1.43 ; 95 \% \mathrm{CI}: 1.02-2.00 ; p=0.04)$. Further, the number of years smoking was also statistically significant in this model. Those who had been smoking longer were less likely to be abstinent at the 7-month follow-up (OR $=0.97 ; 95 \%$ CI: $0.95-0.99 ; p<0.01$ ).

We also tested NRT use as a predictor of heavy drinking rates at the 7-month follow-up. As presented in Table 2, 


\section{Table 2}

7-day point prevalence abstinence at the 7-month follow-up

\begin{tabular}{lllll}
\hline Outcome & $\begin{array}{l}\text { Used All NRT } \\
\text { (No. Abstinent/No. in Sample) }\end{array}$ & $\begin{array}{l}\text { Used Some NRT } \\
\text { (No. Abstinent/No. in Sample) }\end{array}$ & $\begin{array}{l}\text { Used No NRT } \\
\text { (No. Abstinent/No. in Sample) }\end{array}$ & OR [95\% Cl] \\
\hline Smoking & $30.8 \%(138 / 448)$ & $17.8 \%(61 / 342)$ & $17.0 \%(9 / 53)$ & $2.07[1.46-2.93]^{*}$ \\
Heavy drinking & $78.8 \%(349 / 443)$ & $66.3 \%(226 / 341)$ & $54.7 \%(29 / 53)$ & $2.39[1.28-4.44]^{* *}$ \\
\hline
\end{tabular}

Note. $O R=$ odds ratio; $\mathrm{Cl}=$ confidence interval; tregression model for all NRT compared to some NRT; ${ }^{*} p<0.01 ;{ }^{* *} p=0.01$.

\section{Table 3}

Self-reported reasons for NRT non-use

\begin{tabular}{ll}
\hline Responses & Number \\
\hline Concerned about side effects/health problems & 15 \\
Not ready to quit smoking & 10 \\
Quit without them & 7 \\
Saving for future quit attempt & 7 \\
Do not need products to quit smoking & 3 \\
Dislike having to use products to quit smoking & 2 \\
Thought it wouldn't be useful & 1 \\
Gave them away & 1 \\
Concerned about continued addiction to nicotine & 1 \\
Started smoking again & 1 \\
I do not know & 8 \\
\hline
\end{tabular}

Note. Participants could report more than 1 reason.

NRT use was associated with increased odds of no heavy drinking days $(\mathrm{OR}=2.39,95 \% \mathrm{CI}: 1.28-4.44 ; p=0.01)$. Post-hoc probing revealed those who used all of their NRT were more likely to report no heavy drinking days than those who used some or none ( $78.8 \%$ vs. $66.3 \%$ and $54.7 \%$; $p$ 's $<0.01)$. Treatment group status was non-significant in this model ( $p=0.15)$, as was completion of the second counselling session $(p=0.35)$. The percentage of heavy drinking days at baseline was also significant in this model, suggesting that those who had a higher number of heavy drinking days at baseline were more likely to report heavy drinking days in the past week at the 7-month follow-up $(\mathrm{OR}=0.07$; 95\% CI: $0.04-0.14 ; p<0.01)$.

\section{Exploratory Analyses - Reasons for NRT Non-Use}

Participants who reported not using the mailed NRT dose $(N=53)$ were asked to self-report their reason for nonuse. Table 3 presents a frequency count for reasons for NRT non-use. The most frequently reported reasons for not using NRT were: concerned about side effects/health problems, not ready to quit smoking, quit without using the medication, and saving the medication for a future quit attempt.

\section{Discussion}

The results suggest that NRT use among heavy drinking smokers calling a quitline could improve both smoking quit rates and alcohol use reduction. The vast majority of participants reported using the medication (93.7\%), but quit rates and alcohol use reduction differed for those who used all of the 2 week supply of NRT as opposed to only using some of the medication. Those heavy drinking smokers who reported using all of the free NRT sent to them were more likely to report smoking abstinence at the 7-month follow-up compared to those who used only a portion of the NRT dose. Further, those with greater NRT adherence were also more likely to report no heavy drinking days compared to those who used less.

These findings are consistent with previous studies of NRT use (even at short durations) and smoking quit rates. In another study, evaluating use of a 2-week supply of NRT, $78 \%-89 \%$ of smokers who received NRT reported use of the medication, and the patches were used for an average of 9 days (Cummings et al., 2006). This is similar to the $93.7 \%$ found in the present study. Further, in a randomised clinical trial evaluating NRT sampling (i.e., providing a 2-week starter pack to participants), $73 \%$ of smokers used the product, and average duration of use was 9 days (Carpenter et al., 2011). Further, those who received the NRT to sample were more likely to make a quit attempt. In a study of 204 smokers using an active nicotine patch, $68 \%$ of participants used the patch for $\geq 20$ days, and the odds of abstinence were higher for this group compared to those who used the patch less than 20 days (Shiffman et al., 2008). The present study adds to the extant literature by suggesting that a majority of heavy drinking callers to a state quitline who receive a starter pack of NRT will use the medication, and greater NRT use was associated with improved long-term smoking cessation rates.

Group assignment and completion of the second counselling call independently contributed to smoking outcomes such that those who received more of the alcohol and tobacco counselling were more likely to be abstinent at the 7-month follow-up. These variables did not contribute to drinking outcomes, but those who used all of their NRT were more likely to report no heavy drinking days. In other words, regardless of what type of counselling they received, those who used the 2-week of supply of NRT were more likely to have no heavy drinking days after 7 months. The data from the present study do not allow for an analysis of potential mechanisms responsible for this finding, but previous data suggest that NRT may affect subjective and physiological alcohol responses. A laboratory study with heavy-drinking 
smokers demonstrated that transdermal nicotine replacement compared to mild deprivation (i.e., placebo patch) delayed the initiation of drinking, and resulted in fewer drinks consumed during the self-administration period (McKee, O’Malley, Shi, Mase, \& Krishnan-Sarin, 2008). In addition, smoking and drinking are known to have a very close behavioural link (McKee, Falba, O'Malley, Sindelar, \& O'Connor, 2007; Shiffman \& Balbanis, 1996). If participants were likely to smoke and drink simultaneously, then the reduction in drinking may be a result of reduction in smoking following NRT use. An alternative explanation might be self-selection bias, with those most committed to stopping smoking more likely to use the NRT and also to avoid drinking alcohol. Regardless, adherence to NRT use appears to be a meaningful predictor of long term smoking abstinence and reduced alcohol use among a heavy drinking group of smokers. It is also possible that smoking and drinking outcomes could be improved upon by ensuring greater adherence to NRT in heavy-drinking smokers who elect to use pharmacotherapy.

The 2 most endorsed reasons for NRT non-use were (1) not ready to quit smoking and (2), concerned about side effects/health problems. Therefore, perhaps efforts to improve medication use could be directed towards brief motivational interviewing to improve readiness to quit. In addition, describing all potential side effects of NRT and describing methods to mitigate side effects may help to assure participants about the safety of NRT products. Participants should also be made aware of the new changes suggested by the FDA for NRT labelling (US Food and Drug Administration, 2013). The warning to not use NRT while smoking was removed, and NRT use for a prequit time period may promote initial smoking cessation (Fucito et al., 2014). quitlines currently follow the former packaging restrictions in their directions for NRT use and recommend that quitline callers cease NRT use if they smoke. The increased flexibility for usage under the FDA label changes may promote cessation even in the instance of a lapse, and make participants feel more comfortable when using NRT.

One possible limitation of this analysis is that we are unable to assess which came first: medication discontinuation/suboptimal use or relapse to smoking. Unfortunately, the nature of the dataset does not allow us to pinpoint the time at which these events may have occurred. We are also unable to assess reason for discontinuation for those who reported using the medication. It is possible that NRT may not be effective for those who ceased use, or participants may have relapsed prior to discontinuation. There may be a more complex relationship among amount of NRT use, initial cessation, and long-term smoking abstinence than we are able to assess. Another limitation of this study is the lack of systematic assessment of NRT use. While participants were asked to categorise their NRT use (e.g., used about half), attempts to capture more data (e.g., used 6 out of 14 patches) or follow-ups on appropriate NRT use (e.g., Did you always wear your patch for a full
24 hours?) would allow for more fine-grained analyses of treatment outcomes. Further, the data collection tool used at the 7-month follow-up only asked for reasons for non-use from those who reported using none of the NRT. However, collecting this data from the participants who discontinued use would assist in elucidating the reasons for NRT discontinuation. The $43 \%$ response rate at the 7-month follow-up is also a limitation of the study, and it will be important for future quitline studies to strive to improve follow-up rates. Finally, the use of self-report data for smoking cessation status may have been a limitation in this study.

In conclusion, many heavy drinking smokers calling the quitline used the 2-week supply of NRT. Using the complete 2-week supply of NRT and receiving alcohol and tobacco counselling may improve smoking quit rates and reductions in heavy drinking. quitlines are a low-cost, easily accessible treatment option for smokers interested in quitting. Efforts to improve NRT adherence and alcohol education among quitline callers who drink heavily may have a significant effect on smoking and drinking outcomes.

\section{Financial Support}

This research was supported by NIH grants R01CA140256 (to Dr Toll), T32-DA007238 (to Dr Rojewski), and P30 CA138313 (Hollings Cancer Centre Support grant which provides support to Dr Cummings). Its contents are solely the responsibility of the authors and do not necessarily represent the official view of NIH.

\section{Conflict of Interest}

Dr Cummings provides expert testimony in litigation against cigarette manufacturers, provides consulting advice and has received grants from Pfizer, and previously served as a co-investigator on a multi-centre trial evaluating a nicotine vaccine from Nabi Biopharmaceuticals. Dr Toll is the recipient of a grant for medicine only from Pfizer Pharmaceuticals.

\section{Ethical Standards}

The authors assert that all procedures contributing to this work comply with the ethical standards of the relevant national and institutional committees on human experimentation and with the Helsinki Declaration of 1975, as revised in 2008.

\section{References}

Balmford, J., Borland, R., Hammond, D., \& Cummings, K. M. (2011). Adherence to and reasons for premature discontinuation from stop-smoking medications: Data from the ITC four-country survey. Nicotine \& Tobacco Research, 13(2), 94102. doi:10.1093/ntr/ntq215.

Banta, J. E., Haskard, K. B., Haviland, M. G., Williams, S. L., Werner, L. S., Anderson, D. L. et al. (2009). Mental health, binge drinking, and antihypertension medication 
adherence. American Journal of Health Behavior, 33(2), 158171. doi:10.5993/AJHB.33.2.5.

Braithwaite, R. S., McGinnis, K. A., Conigliaro, J., Maisto, S. A., Crystal, S., Day, N. et al. (2005). A temporal and dose-response association between alcohol consumption and medication adherence among veterans in care. Alcoholism: Clinical and Experimental Research, 29(7), 11901197. doi:10.1097/01.ALC.0000171937.87731.28.

Burns, E. K., \& Levinson, A. H. (2008). Discontinuation of nicotine replacement therapy among smoking-cessation attempters. American Journal of Preventive Medicine, 34(3), 212-215. doi:10.1016/j.amepre.2007.11.010.

Cahill, K., Stevens, S., \& Lancaster, T. (2014). Pharmacological treatments for smoking cessation. JAMA, 311(2), 193-194. doi:10.1001/jama.2013.283787.

Carpenter, M. J., Hughes, J. R., Gray, K. M., Wahlquist, A. E., Saladin, M. E. et al. (2011). Nicotine therapy sampling to induce quit attempts among smokers unmotivated to quit: A randomized clinical trial. Archives of Internal Medicine, 171, 1901-1907.

Cummings, K. M., Fix, B., Celestino, P., Carlin-Menter, S., O'Connor, R., \& Hyland, A. (2006). Reach, efficacy, and costeffectiveness of free nicotine medication giveaway programs. Journal of Public Health Management and Practice, 12(1), 3743. Retrieved from http://journals.lww.com/jphmp/Fulltext/ 2006/01000/Reach,_Efficacy,_and_Cost_effectiveness_of_ Free.9.aspx.

Fiore, M. C., Schroeder, S. A., \& Baker, T. B. (2014). Smoke, the chief killer - Strategies for targeting combustible tobacco use. New England Journal of Medicine, 370(4), 297299. doi:10.1056/NEJMp1314942.

Fucito, L. M., Bars, M. P., Forray, A., Rojewski, A. M., Shiffman, S., Selby, P. et al. (2014). Addressing the evidence for FDA nicotine replacement therapy label changes: A policy statement of the association for the treatment of tobacco use and dependence and the society for research on nicotine and tobacco. Nicotine \& Tobacco Research, 16(7), 909-914. doi:10.1093/ntr/ntu087.

IBM. (2013). IBM SPSS Statistics for Windows, Version 22.0. Armonk, NY: IBM Corp.

McKee, S. A., Falba, T., O’Malley, S. S., Sindelar, J., \& O’Connor, P. G. (2007). Smoking status as a clinical indicator for alcohol misuse in us adults. Archives of Internal Medicine, 167(7), 716-721. doi:10.1001/archinte.167.7.716.

McKee, S. A., O’Malley, S. S., Shi, J., Mase, T., \& KrishnanSarin, S. (2008). Effect of transdermal nicotine replacement on alcohol responses and alcohol selfadministration. Psychopharmacology, 196(2), 189-200. doi:10.1007/s00213-007-0952-3.

National Institute on Alcohol Abuse Alcoholism. (2005). Helping patients who drink too much: A clinician's guide (Vol. NIH Publication No. 05-3769). MD: National Institutes of Health Bethesda.

Shiffman, S., \& Balbanis, M. (1996). Do drinking and smoking go together?. Alcohol Health and Research World, 20(2), 107110.

Shiffman, S., Sweeney, C. T., Ferguson, S. G., Sembower, M. A., \& Gitchell, J. G. (2008). Relationship between adherence to daily nicotine patch use and treatment efficacy: Secondary analysis of a 10 week randomized, double-blind, placebocontrolled clinical trial simulating over-the-counter use in adult smokers. Clinical Therapeutics, 30(10), 1852-1858. doi:10.1016/j.clinthera.2008.09.016.

Toll, B. A., Martino, S., O’Malley, S. S., Fucito, L. M., McKee, S. A., Kahler, C. W. et al. (2015). A randomized trial for hazardous drinking and smoking cessation for callers to a quitline. Journal of Consulting and Clinical Psychology, 83(3), 445-454. doi:10.1037/a0038183.

US Food and Drug Administration. (2013). Nicotine replacement therapy labels may change. FDA Consumer Health Information. Silver Spring, MD: U. S. Department of Health and Human Services. 